\section{SOI: $1.1 /$ TAS DOI: $10.15863 /$ TAS International Scientific Journal Theoretical \& Applied Science}

p-ISSN: 2308-4944 (print) e-ISSN: 2409-0085 (online)

Year: 2018 Issue: $06 \quad$ Volume: 62

Published: $26.06 .2018 \quad$ http://T-Science.org

\section{S. U. Zhanatauov} candidate of physics and mathematical sciences, Department «Iinformation technologies, mathematics, physics», Associate professor, Noncommercial joint-stock company "Kazakh national agrarian university" Kazakhstan sapagtu@mail.ru

SECTION 2. Applied mathematics. Mathematical modeling.

\title{
MODEL OF DIGITALIZATION OF INDICATORS OF INDIVIDUAL CONSCIOUSNESS
}

Abstract: In the article, using the language of the description of situations " attribute -name -value-unit of measurement". a mathematical model of digitization of indicators of individual consciousness of the individual is developed. Cognitive analysis and modeling were carried out on the basis of the Inverse Model of the Principal Component Analysis [11]. An illustrative example is given of giving names to 6 correlated indicators of individual consciousness with given 4 latent factors of individual consciousness.

Key words: latent factors exceeding the threshold of moderate manifestation (perception by the individual) correlated indicators of the individual consciousness of the individual.

Language: Russian

Citation: Zhanatauov SU (2018) MODEL OF DIGITALIZATION OF INDICATORS OF INDIVIDUAL CONSCIOUSNESS. ISJ Theoretical \& Applied Science, 06 (62): 101-110.

Soi: $\underline{\text { http://s-o-i.org/1.1/TAS-06-62-20 Doi: crossef https://dx.doi.org/10.15863/TAS.2018.06.62.20 }}$

\section{МОДЕЛЬ ЦИФРОВИЗАЦИИ ПОКАЗАТЕЛЕЙ ИНДИВИДУАЛЬНОГО СОЗНАНИЯ}

Аннотация: В статье с применением языка описания ситуаций «показатель- наименованиезначение-единияа измерения». разработана математическая модель ичифровизации показателей индивидуального сознания индивида. Когнитивное анализ и моделирование проводились на основе Обратной Модели Главных Компонент. Приведен иллюстратиный пример придания названий 6 коррелированным показателям индивидуального сознания при заданных 4 скрытых факторах индивидуального сознания.

Ключевые слова: скрытые факторы, превышающие порог умеренного проявления (восприятия индивидом) коррелированные показатели индивидуального сознания индивида

\section{Введение.}

На индивиды любой страны влияют разнородные факторы, воздейстующие на его поведение, принятие решений в повседневной жизни, в быту, в бизнесе, в проявлениях социальной активности.

Общественный прогресс все быстрее изменяет индивидуальное сознание. В социальных группах существуют многочисленные маркеры «свой-чужой», связанные с раздельным проживанием «людей на красивых машинах» и быдла, которое «не должно сметь своё суждение иметь». Аналогичных факторов, ситуаций проявления или осуждения социального расизма во всех его проявлениях мы узнаем часто из СМИ. Государственные программмы модернизации общественного сознания актуализируют проблему, особо подчеркивая, что «занять место в передовой группе, сохраняя прежнюю модель сознания и мышления, невозможно» [1]. Новые отношения «власть-гражданское общество», модернизация индивидуального и общественного сознания актуализируют вопросы формализации предметной области «индивидуальное сознание», рассматриваемой в данной статье. Мы считаем не достаточыми обличительные сообщения, заметки «борцов с коррупциией», поражающих нас как своим размахом, так и неэффективностью, невольно принуждающей нас к беспокойству, к смирению, к преодолению страха. К индивиду (например, в процессе менеджмента предприятия где есть АСУ «поддержка принятия решений») изредка или часто применяют меры принуждения, вынуждающих индивида прибегать к некоторому обману «в интересах 
дела». Существуют разные способы профилактики, борьбы с такими проявлениями. Но обличительный задор борцов всегда направлен на личности, они вызывают у индивида страх, стремление обмануть «систему менеджмента «shnaiy ondru-kondru-aldaukorkytu».

Групп факторов, которых можно назвать «главными», определяющими существует очень много. Рассмотрим те факторы, которые в последнее время болезненно воспринимаются на бытовом уровне («на кухне»). Среди мнений, «проясняющих» нашу действительность, существуют такие как «если бог даст, то...», «жизнь наша - театр», «кругом обман и ...», «...заставят-никуда не денешься». Даже в поэзии звучат слова про угрозы, устрашения, запугивания «...бессердечных и бездушных что запугали чем-то полстраны» ( https://www.inpearls.ru/). С телеэкранов в сюжетах фильмов разного жанра отчетливо внушаются существование не столько добра и зла, но и «менеджмент» их способов проявлений. Та или иная ложь выдается за правду. Затронутые ассоциации, верования, а также технологии достижения целей исследованы и доступны нучному сообществу[2-9].

Актуально иметь модели, раскрывающие в той или иной степени, суть социального явления. Слишком мало встречается попыток количественного измерения рассматриваемых ниже факторов, их взаимосвязей с измеряемыми социальными, экономическими, индивидуальными или с другими показателями. В текстах [2-9] изучаются смысловые, специальные, измеряемые факторы. Широко представлены зависимости между политическими, экономическими, социальными, индивидуальными свойствами, факторами, событиями. Но мало разработок по формализации [2-9].

Рассмотрим легко воспринимаемые индивидом скрытые факторы. Еще раз подчеркнем -мы утверждаем, что рассматриваемые факторы являются одними из многих, не являются определяющими в жизни общества. Будем считать источником проявлений 3-х из 4-х рассматриваемых факторов наличие дисбалансов между возможностями и потребностями, между возможностями и ответственностью.

\section{Скрытые социально-экономические факторы}

Известно, что существуют скрытые факторы, значения которых не измеряются, но они вычисляются как значения переменных, равных линейным комбинациям фактически измеряемых показателей. Например, в работе [10] применяемые нами ниже главные компоненты (principal components) называют «смысловыми» (semantic variables). Смысл их передается в фразах «статус родителей», «средняя школьная оценка за устную речь». Другие фразы, передающие иные смыслы, приведены, например, в работах [11-14]. Число таких фраз равно $\ell=2,3,4$. Наши $\ell<$ n главные компоненты будем интерпретировать как главные содержательно имеющие экономический или иной смысл факторы. Им поставим в соответствие $\ell$ переменные. В модели число у-переменных полагаем равным числу $\mathrm{n}$ измеряемых показателей (представленных в модели через n zпеременные) реального объекта, но будем придавать названия только тем $\mathrm{z}$-переменным, которые имеют веса, превышающие порог «умеренного» проявления (восприятия индивидом) коррелированных показателей индивидуального сознания индивида.

В нашей модели будем учитывать значения весов $\ell=4$ факторов $\lambda_{1}, \ldots, \lambda_{4}$ (значения только $\ell$ из $\mathrm{n}$ y-переменных), будем определять названия, значения $\mathrm{n}$ коррелированных $\mathrm{z}$-переменных $\mathrm{Z}_{1}, \ldots, \mathrm{Z}_{\mathrm{n}}$. Эту задачу схематично изобразим так: $\Lambda_{\ell \ell}=>\left(\mathrm{y}_{1} \mathrm{y}_{2}, \ldots, \mathrm{y}_{\ell}\right)=>\left(\mathrm{R}_{\mathrm{nn}}, \mathrm{C}_{\mathrm{nn}}, \mathrm{Z}_{\mathrm{mn}}\right)$. Для численного моделирования матрицы «весов» $\mathrm{C}_{\mathrm{nn}}$, матрицы безразмерных значений $\mathrm{Z}$-переменных $Z_{\mathrm{mn}}$ будем применять ОМ ГК [11], апробированная в других предметных областях [12-14].

Разработаем математическую модель цифровизации показателей индивидуального сознания для предметной области «индивидуальное сознание» (ИС).

Одним из примеров выявления факторов, скрываемых за измеряемыми показателями в школе у школьников и у родителей, является пример, опубликованный в статье [12]. Когнитивная карта и модель социальноэкономических факторов карьерной успешности школьников муниципальных школ США позволили «вытянуть» содержательный вывод из данных. Обоснованность формализации в предметной области подтверждена двумя фразами для факторов, приводимых ниже. Схема изображается в виде ПМГК: $\mathrm{Z}_{\mathrm{mn}}=>\left(\mathrm{R}_{\mathrm{nn}}, \mathrm{C}_{\mathrm{nn}}, \Lambda_{\mathrm{nn}}, \mathrm{Y}_{\mathrm{mn}}\right)$. Ей соответствует прямая смысловая схема: (смысл $\left(\mathrm{z}_{1}\right), \ldots$, смысл $\left(\mathrm{z}_{\mathrm{n}}\right)$ =>

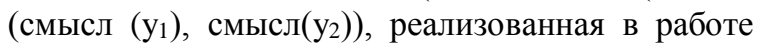
[12]. Ниже с применением ОМ ГК реализована смысловая схема, обратная к приведенной выше: (смысл $\quad\left(\mathrm{y}_{1}\right), \quad$ смысл $\left.\left(\mathrm{y}_{2}\right)\right)=>\quad\left(\right.$ смысл $\left(\mathrm{z}_{1}\right), \ldots$, смысл $\left(\mathrm{z}_{\mathrm{n}}\right)$.

При реализации прямой смысловой схемы были решены задачи по выявляению $\ell=2$ фактора (первые 2 столбца матрицы $\mathrm{Y}_{\mathrm{mn}}$ содержат $\mathrm{m}$ значений $\mathrm{y}$-переменных $\left.\mathrm{y}_{1}, \mathrm{y}_{2}\right)$, влияющих на будущую карьерную успешность школьника 
(целевой содержательный критерий). Остальные $\mathrm{n}-\ell$ столбцов не рассматриваются, их дисперсии малы, интерпртируются как несущественные факторы системы. Элементы спектра $\Lambda_{\mathrm{nn}}=\operatorname{diag}\left(\lambda_{1}\right.$, $\left.\ldots, \lambda_{n}\right)$, равны дисперсиям факторов. Но мы рассматриваем только существенные $\lambda_{1}, \ldots, \lambda_{\ell}, \ell=2$.

Но в предметных областях «работают» разные целевые критерии. Поэтому мы будем использовать только формальные уравнения из модели (из ПМ АГК), но будем решать обратную задачу к задаче, решенной в ОМ АГК: $\left(\mathrm{y}_{1}, \mathrm{y}_{2}, \mathrm{y}_{3}, \mathrm{y}_{4}\right)$ $=>Z_{\mathrm{mn}}$. Здесь $Z_{\mathrm{mn}}$-таблица (матрица) размерности m-на-n, где n - число моделируемых нами показателей. Они адекватны реальным измеряемым показателям, характеризующих рассматриваемую систему \{«правильно», «принудительно», «с обманом», «под страхом»\}. Фиксированные значения дисперсий от значений этих 4 факторов обозначим как $\lambda_{1}, \ldots, \lambda_{4}$. Соответствующие параметры, переменные, уравнения ограничений, целевая функция разработаны, опубликованы в [11-14].

Для реализации нашей модели необходимо реализовать схему: $\left(\lambda_{1}, \ldots, \lambda_{\mathrm{n}}\right) \Rightarrow\left(\mathrm{y}_{1}, \mathrm{y}_{2}, \mathrm{y}_{3}, \mathrm{y}_{4}\right)$ $\Rightarrow Z_{\mathrm{mn}}$. Суть этой модели - в выборе 4-х главных факторов, определяющих $\mathrm{m}>\mathrm{n}$ значений $\mathrm{n}>4$ коррелированных показателей, характеризующих нашу систему \{«правильно», «принудительно», «с обманом», «под страхом»\}. Возможно, что каждый элемент нашей системы характеризуется разными числами показателей, например, $\mathrm{n}=5+9+7+4=25$.

Рассмотрим систему из четырех некоррелированных у-переменных $\mathrm{y}_{1}, \mathrm{y}_{2}, \mathrm{y}_{3}, \mathrm{y}_{4}$. Им в соответствие поставим 4 фактора проявлений индивидуального сознания, их проявления полагаем независимыми. Поставим в соответствие этим 4 неизмеряемым показателям (обобщенным факторам) теоретические случайные величины $\xi_{1}, \xi_{2}, \xi_{3}, \xi_{4}$. Это позволит нам формализовать динамику, взаимосвязи, присущие реальным значениям наших 4 факторов.

Будем рассматривать безразмерные значения всех анализируемых переменных, включая переменные $\mathrm{y}_{1}, \mathrm{y}_{2}, \mathrm{y}_{3}, \mathrm{y}_{4}$ и переменные (назовем их Z-переменные), образующие линейные комбинации $\mathrm{y}_{1}=\mathrm{z}_{1} \mathrm{c}_{11}+\ldots+\mathrm{z}_{\mathrm{n}} \mathrm{c}_{\mathrm{n} 1}, \mathrm{y}_{2}=\mathrm{z}_{1} \mathrm{c}_{13}+\ldots+\mathrm{z}_{\mathrm{n}} \mathrm{c}_{\mathrm{n} 3}$, $\mathrm{y}_{3}=\mathrm{z}_{1} \mathrm{c}_{13}+\ldots+\mathrm{z}_{\mathrm{n}} \mathrm{c}_{\mathrm{n} 3} ., \quad \mathrm{y}_{4}=\mathrm{z}_{1} \mathrm{c}_{14}+\ldots+\mathrm{z}_{\mathrm{n}} \mathrm{c}_{\mathrm{n} 4} . \quad$ Значения остальных модельных факторов у-переменных $\mathrm{y}_{5}, \ldots, \mathrm{y}_{\mathrm{n}}$ моделируются, их значения удовлетворяют соотношениям $\mathrm{y}_{\mathrm{j}}=\mathrm{z}_{1} \mathrm{c}_{1 \mathrm{j}}+\ldots$ $+\mathrm{Z}_{\mathrm{n}} \mathrm{c}_{\mathrm{nj}}, \mathrm{j}=5, \ldots, \mathrm{n}$, но их дисперсии пренебрежимо малы $\lambda_{4}<\lambda_{0}, \ldots, \lambda_{n}<\lambda_{0}$, причем значение $\lambda_{0}$ удовлетворяет критерию приблизительного равенства нулю дисперсий у -переменных $\mathrm{y}_{5}, \ldots, \mathrm{y}_{\mathrm{n}}$.
Это означает с точки зрения математической статистики равенство нулю парных коэффициентов корреляции: $\operatorname{corr}\left(\mathrm{z}_{1}, \mathrm{y}_{2}\right)=\mathrm{c}_{12}=0$, $\operatorname{corr}\left(\mathrm{z}_{1}, \mathrm{y}_{3}\right)=\mathrm{c}_{13}=0, \quad$ corr $\quad\left(\mathrm{z}_{2}, \mathrm{y}_{3}\right)=\mathrm{c}_{23}=0$. Доказательство факта о том, что матрица $\mathrm{C}_{66}$ собственных векторов является несимметричной корреляционной матрицей коэффициентов парной корреляции (y,z)-переменных, имеются в [15-17].

\section{Нахождение смыслов скрытых социально-экономических факторов}

Первый фактор (например, «статус родителей») имеет наибольшую дисперсию $\lambda_{1}$ [12]. Соответствующие ему значения будем выражать через значения 1-ой у-переменной $\mathrm{y}_{1} \mathrm{c}$ наибольшей изменчивостью-дисперсией $\lambda_{1}$. Этим мы подтверждаем, что у-переменная у1 является фактором наибольшей важности. Она (упеременная) равна линейной комбинации $\mathrm{n} \mathrm{z}$ переменных, среди которых только некоторые $\left(\mathrm{z}_{1}, \mathrm{z}_{4}\right)$ имеют «ощутимые» веса, а остальные пренебрежимо малые, т.е. обнуляемые при «осмыслении» их веса. Веса $\mathrm{c}_{\mathrm{i} 1}=-0.6548 \mathrm{z}_{\mathrm{i} 1}$ ci4=$0.7014 z_{i 4}$ принадлежат интервалу «заметных» корреляций. Все значения из этого интервала, если они являются коэффициентами уравнения, будут участвовать в процессе нахождения вышенайденного смысла переменной $\mathrm{y}_{1}$. Бесконечное множество пар весов из указанного интервала придадут один и тот же смысл переменной $\mathrm{y}_{1}$. Если $\mathrm{y}_{1}=\mathrm{z}_{1} \mathrm{c}_{11}+\ldots+\mathrm{z}_{\mathrm{n}} \mathrm{c}_{\mathrm{n} 1}$, $\mathrm{y}_{2}=\mathrm{z}_{1} \mathrm{c}_{13}+\ldots+\mathrm{z}_{\mathrm{n}} \mathrm{c}_{\mathrm{n} 3}, \quad \mathrm{y}_{3}=\mathrm{z}_{1} \mathrm{c}_{13}+\ldots+\mathrm{z}_{\mathrm{n}} \mathrm{c}_{\mathrm{n} 3}$. то смысл $\left(\mathrm{y}_{2}\right)=\mathrm{c}_{12} \times$ смысл $\left(\mathrm{z}_{1}\right)+\mathrm{c}_{42} \times$ смысл $\left(\mathrm{z}_{4}\right) . \quad$ Здесь математической модель выражается через упеременную в виде функции вида $\mathrm{y}_{\mathrm{i} 2}=-0.6548 \mathrm{z}_{\mathrm{i} 1}$ $0.7014 z_{i 4}+\varepsilon_{2}$. Смысл новой переменной №2 равен сумме зависимых между собой смыслов: смысл 1 и смысл 2, где смысл $\left(\mathrm{z}_{\mathrm{i} 1}\right)=$ =«Оплата штатного школьного персонала» и смысл $\left(\mathrm{z}_{\mathrm{i} 4}\right)=$ исредняя школьная оценка за устную речь» выразим фразой, состоящее из 2 частей. Ссмысл $1=$ =«стимулированное оплатой (производимой школой) влияние штатных учителей и смысл 2=«профессональное влияние учителей, оценивших успеваемость учеников в баллах». Этими фразами мы описываем другие события и факты из той же многослойной проблемной области, но другими словами. Из линейной комбинации - суммы смыслов, формируем результирующий смысл смысл $\left(\mathrm{y}_{\mathrm{i} 2}\right) \approx-$ $0.6548 *$ (смысл_1) $-0.7014 *($ смысл_2) $=-0.6548$

*(оплата школьного персонала в расчете на одного школьника)-0.7014*(средняя школьная оценка за устную речь) $\rightarrow-1 *$ (*(оплата школьного персонала в расчете на одного школьника)-1*(средняя школьная оценка за устную речь). Так как «оплата школьного 
персонала в расчете на одного школьника» не может быть реализована без выставления оценок, то «оплата» служит фоном для выставления в журналах «средней школьной оценки за устную речь». Определяющей фразой является «средняя школьная оценка за устную речь». Так как 1-ая часть - «оплата школьного персонала в расчете на одного школьника», влияет на 2-ую часть, способствует реализации событий - эзаменов, результатом которых является появление в журналах средней оценки, то результирующим событием является «средняя школьная оценка за устную речь», и нашими целевыми факторами являются факторы ученика, а не факторы родителей, и не факторы школы, то в качестве результирующего смысла у-переменной у $_{2}$ назначаем смысл 2-ой части- «средняя школьная оценка за устную речь». Имеем равенство смысл(у)= смысл_1+смысл_2.

Мы сформулировали содержательный смысл новой у-переменной №2: «стимулированное зарплатой влияние штатных учителей профессионалов, оценивших успеваемость учеников в баллах». Мы использовали значения «баллов», приписываемых значениям Zпеременной №4. При использовании другой переменной с другой единицей измерения смысл z-переменной №4 передавался бы другими фразами. Коротко смысл новой у-переменной №2 звучит так: «средняя школьная оценка за устную речь».

Этот фактор успешной карьеры «измеренная» посредством вычисленных значений $у$-переменной №2 $\mathrm{y}_{2}$ содержит $\left.\lambda_{2}=1.3953 / 6\right) * 100 \%=0.23255 * 100 \% \quad=23.255 \%$ информации, получившей конкретный смысл [12]. В итоге $43 \%+23.255 \%=83.485 \%$ информации превращены в конкретные «цифровые» знания.

Опыт, приобретенный в этом примере, в других предметных областях пригоден и в нашем рассматриваемом случае. Сперва рассмотрим социально-экономические факторы, затем факторы индивидуального сознания Термин «главная компонента» относится прямой модели анализа главных компонент (principal component analysis [18]).

\section{Главные скрытые факторы индивидуального сознания}

Рассмотрим один из простых примеров моделирования, например, 4 скрытых обобщенных факторов индивидуального сознания, которые существенно влияют на индивиды, не подверженные влиянию социального расизма, в обществе. Назовем их обобщенно (shnaiy ondru, kondru, aldau, korkytu). В казахскоязычной среде эти термины легко воспринмаются и ассоциируются однозначно. Вопросы словесного выражения и восприятия фраз сложны, ибо каждый язык «по своему хорош», мы не претендуем ни на полноту их охвата, ни на окончательность выводов. Перевод на русский язык не сможет отразить указанных ассоциаций, даже скрупулезный подбор слов не сможет помочь. «Правильнее будет так...» часто звучащие фразы при дискуссиях. Но сделаем хоть плохой, но перевод. Этой четверке слов соответствуют на русском языке слова «по правде (правильно) поступать», «принудить к...», «обмануть, чтобы...», «страху нагнать...».

Пусть $\lambda_{1} / \mathrm{n}, \ldots, \lambda_{\mathrm{n}} / \mathrm{n}$ - веса наших факторов, где элементы суммы подчиняются условию нормировки $\lambda_{1} / \mathrm{n}+\ldots+\lambda_{\mathrm{n}} / \mathrm{n}=1$ для фиксации в $\%$-ах доли каждого слагаемого. Мы выше зафиксировали $\ell=4<\mathrm{n}$. Число $\mathrm{n}$ факторов должно быть больше числа $\ell$ скрытых обобщенных факторов, смыслы которых считаем известными. Пока мы рассмотрели $\ell=4$ таких факторов. Элементы $\lambda_{1}, \ldots, \lambda_{\mathrm{n}}$, удовлетворяющие условию $\lambda_{1}+\ldots+\lambda_{4}=\mathrm{f}_{4} * \mathrm{n}$, где $\mathrm{f}_{4}-$ доля суммы 4-х элементов $\lambda_{1}, \ldots, \lambda_{4} \quad$ в сумме $\mathrm{n}$ элементов $\lambda_{1}+\ldots+\lambda_{\mathrm{n}}=\mathrm{n}$. Элементы $\lambda_{1}, \ldots, \lambda_{\mathrm{n}}$ являются параметрами из другой модели [11]. Они являются элементами спектра $\Lambda_{\mathrm{nn}}=\operatorname{diag}\left(\lambda_{1}, \ldots, \lambda_{n}\right)$. Далее в отличие от модели из [12] спектр $\Lambda_{\mathrm{nn}}=\operatorname{diag}\left(\lambda_{1}, \ldots, \lambda_{\mathrm{n}}\right)$ неизвестной корреляционной матрицы $\mathrm{R}_{\mathrm{nn}}$ назначим входным объектом ОМ ГК: $\Lambda_{\mathrm{nn}}=>\left(\mathrm{C}^{(\ell)}{ }_{\mathrm{nn}}\right.$, $\left.\mathrm{R}^{(\ell)}{ }_{n n}, \mathrm{Y}^{(\mathrm{t})}{ }_{\mathrm{m}, \mathrm{n}}, \mathrm{Z}^{(\mathrm{t}, \ell)}{ }_{\mathrm{m}, \mathrm{n}}\right), \mathrm{t}=1, \ldots \mathrm{k}_{\mathrm{t}}, \ell=1, \ldots, \mathrm{k}_{\ell}$. Элементы $\mathrm{R}_{\mathrm{nn}}, \mathrm{C}_{\mathrm{nn}}, \Lambda_{\mathrm{nn}}, \mathrm{Y}_{\mathrm{mn}}$ прямой модели главных компонент (ПМ ГК) $\mathrm{Z}_{\mathrm{mn}}=>\left(\mathrm{R}_{\mathrm{nn}}, \mathrm{C}_{\mathrm{nn}}, \Lambda_{\mathrm{nn}}, \mathrm{Y}_{\mathrm{mn}}\right)$, применяемые в модели из [12], могут быть элементами и $О$ $\mathrm{Z}^{(\mathrm{t}, \ell)}{ }_{\mathrm{m}, \mathrm{n}}=\mathrm{Y}^{(\mathrm{t})}{ }_{\mathrm{mn}} \mathrm{C}^{(\mathrm{t}, \ell) \mathrm{T}}{ }_{\mathrm{nn}} \quad$ моделируется в нашей модели цифровизации (оцифровки) показателей индивидуального сознания. Интересные свойства данной $\Lambda$-выборки доказаны в $[11,19]$. Пример применения ПМ ГК [12] поможет читателю понять детали применения ОМ ГК в излагаемой модели. В этих моделях применяется (H-Z)модель, достаточно удачно применявшаяся в других предметных областях [11-17].

Рассмотрим нашу систему из четырех факторов. Им соответствуют 4 у-переменные $\mathrm{y}_{1}, \mathrm{y}_{2}, \mathrm{y}_{3}, \mathrm{y}_{4}$, значения которых мы будем моделировать ниже. Число значений в каждой из 4-x y-переменных равно $\mathrm{m}>\mathrm{n}$, соответствует матрица собственных векторов $\mathrm{C}_{66}=\left\{\mathrm{c}_{\mathrm{ij}}\right\}$. Матрице $\mathrm{C}_{66}$ соответствует матрица весов $\mathrm{C}_{66 .}^{2}=\left\{\mathrm{c}^{2}{ }_{\mathrm{ij}}\right\},[12-15] \mathrm{i}=1, \ldots, 6 ; j=1, \ldots, 6$. Элементы $\mathrm{c}_{\mathrm{ij}}$ равны коэффициентам корреляции $\mathrm{c}_{\mathrm{ij}}=\operatorname{corr}\left(\mathrm{y}_{\mathrm{i}}, \mathrm{z}_{\mathrm{j}}\right)$ между i-ой $\mathrm{y}$-переменной и j-ой $\mathrm{z}$-переменной. Значение коэффициента парной корреляции между двумя $\mathrm{z}$-переменными $\mathrm{r}_{\mathrm{ij}}=\operatorname{corr}\left(\mathrm{z}_{\mathrm{i}}, \mathrm{z}_{\mathrm{j}}\right)$ является константой (коэффициентом) линейной связи между значениями двух $\mathrm{z}$-переменных $[11,15]: z_{k j}=r_{i j \times} z_{k j}, k=1, \ldots, m, i=1, \ldots, n, j=1, \ldots, n$. Так 
как $\quad \mathbf{c}_{\mathrm{j}}^{\mathrm{T}} \mathbf{c}_{\mathrm{j}}=1, \mathbf{c}_{\mathrm{j}}=\left(\mathrm{c}_{1 \mathrm{j}}, \ldots, \mathrm{c}_{\mathrm{nj}}\right)^{\mathrm{T}}, \quad \mathrm{c}^{2}{ }_{1 \mathrm{j}}+\ldots,+\mathrm{c}^{2}{ }_{\mathrm{nj}}=1, \quad$ то значения чисел $\mathrm{c}^{2}{ }_{1 \mathrm{j}}, \ldots, \mathrm{c}^{2}{ }_{\mathrm{nj}}$ в сумме равных 1 , являются весами при значениях $\mathrm{z}$-переменных $\mathrm{Z}_{1}, \ldots, \mathrm{Z}_{\mathrm{n}}$. Ниже в критериях 1,2,3 используется степень коррелированности $\mathrm{c}_{\mathrm{ij}}=\operatorname{corr}\left(\mathrm{y}_{\mathrm{i}}, \mathrm{z}_{\mathrm{j}}\right)$, превышающий пороговое значение, a специалистам по индивидуальному сознанию более привычен термин «вес». Поэтому всюду ниже значение $c_{i j}=\operatorname{corr}\left(y_{i}, z_{j}\right)$ будем называть «вес», а значение $\mathrm{c}_{\mathrm{ij}}^{2}-$ вес $\mathrm{i}$-ой $\mathrm{z}-$-переменной $\mathrm{z}_{\mathrm{i}}$.

Введем правила, выраженные условным оператором вида: ЕСЛИ...ТО ..., который словесно и конкретно выразим в виде Правил 0,1, 2. Смысл чисел $\lambda_{1}, \lambda_{2} \lambda_{3}$ и $\lambda_{4}$ при $\mathrm{n}=6$ будет проясняться ниже по мере изложения.

Правило о. Если $\lambda_{1} \geq 1, \lambda_{2} \geq 1, \lambda_{3} \geq 1, \lambda_{4} \geq 1$, то необходимо анализировать 1 -ые 4 столбца матрицы собственных векторов $\mathrm{C}_{66}=\left\{\mathrm{c}_{\mathrm{ij}}\right\}$. Матрице $\mathrm{C}_{66}$ соответствует матрица весов $\mathrm{C}_{66 .}^{2}=\left\{\mathrm{c}^{2}{ }_{\mathrm{ij}}\right\}, \mathrm{i}=1, \ldots, 6 ; j=1, \ldots, 6$.

Правило1. В сумму слагаемых $\mathrm{y}_{\mathrm{i} 1}=\mathrm{z}_{\mathrm{i} 1} \mathrm{c}_{1 \mathrm{j}}+\mathrm{z}_{\mathrm{i} 2} \mathrm{c}_{2 \mathrm{j}}+\ldots+\mathrm{z}_{\mathrm{in}} \mathrm{c}_{\mathrm{nj}}, \mathrm{i}=1, \ldots, \mathrm{m}, \mathrm{j}=1$, включать только то слагаемое, в котором значение $\mathrm{z}-$ переменной имеет значимый вес, т.е. «вес» должен удовлетворять критерию $\left|\mathrm{c}_{\mathrm{kj}}\right| \geq \operatorname{const}(1)=0.48$, если $\mathrm{j}=1$.

Правило 2. В сумму слагаемых $\mathrm{y}_{\mathrm{i} 2}=\mathrm{z}_{\mathrm{i} 1} \mathrm{c}_{12}$ $+\mathrm{z}_{\mathrm{i} 2} \mathrm{c}_{22}+\ldots+\mathrm{z}_{\mathrm{in}} \mathrm{c}_{\mathrm{n} 2}, \mathrm{i}=1, \ldots, \mathrm{m}$, включать только то слагаемое, в котором значение $\mathrm{z}$-переменной имеет значимый вес, т.е. «вес» должен удовлетворять критерию $\left|\mathrm{c}_{\mathrm{kj}}\right| \geq \operatorname{const}(\mathrm{j})=0.65$, если $\mathrm{j}=2$.

Правило3. В сумму слагаемых $\mathrm{y}_{\mathrm{i} 3}=\mathrm{z}_{\mathrm{i} 1} \mathrm{c}_{1 \mathrm{j}}+\mathrm{z}_{\mathrm{i} 2} \mathrm{c}_{2 \mathrm{j}}+\ldots+\mathrm{z}_{\mathrm{in}} \mathrm{c}_{\mathrm{nj}}, \mathrm{i}=1, \ldots, \mathrm{m}, \mathrm{j}=3$, включать только то слагаемое, в котором значение zпеременной имеет значимый вес, т.е. «вес» должен удовлетворять критерию $\left|\mathrm{c}_{\mathrm{kj}}\right| \geq \operatorname{const}(3)=0.48$, если $\mathrm{j}=3$.

Правило 4. В сумму слагаемых $\mathrm{y}_{\mathrm{i} 4}=\mathrm{z}_{\mathrm{i} 1} \mathrm{c}_{14}+\mathrm{z}_{\mathrm{i} 2}$ $\mathrm{c}_{24}+\ldots+\mathrm{Z}_{\mathrm{in}} \mathrm{c}_{\mathrm{n} 4}, \mathrm{i}=1, \ldots, \mathrm{m}$, включать только то слагаемое, в котором значение $\mathrm{z}$-переменной имеет значимый вес, т.е. «вес» должен удовлетворять критерию $\left|c_{\mathrm{kj}}\right| \geq \operatorname{const}(4)=0.65$.

Математической моделью новых смысловых переменных являются функции вида $\mathrm{y}_{\mathrm{ij}}=\mathrm{z}_{\mathrm{i} 1} \mathrm{c}_{1 \mathrm{j}}+$ $\mathrm{z}_{\mathrm{i} 2} \mathrm{c}_{2 \mathrm{j}}+\ldots+\mathrm{z}_{\mathrm{in}} \mathrm{c}_{\mathrm{nj}}, \mathrm{i}=1, \ldots, \mathrm{m}$, которые определяются используемой теоретической моделью [18]: ПМ ГК -как метода вычисления единственной матрицы $\mathrm{Y}_{\mathrm{mn}}$, состоящей из $\mathrm{m}$ значений некоррелированных $\mathrm{n}$ у- переменных с ограничениями на веса $\mathrm{c}_{1 \mathrm{j}}{ }^{2}+\mathrm{c}_{2 \mathrm{j}}{ }^{2}+\ldots+\mathrm{c}_{\mathrm{nj}}{ }^{2}=1$, на компоненты собственных векторов: $c_{11} c_{k 1}+$ $\ldots+\mathrm{c}_{\mathrm{n} 1} \mathrm{c}_{\mathrm{kj}}=0, \mathrm{j} \neq 1, \mathrm{k}=1, \ldots, \mathrm{n}$.

При объяснении, присвоении названия zпеременной используем правило, где ощутимость влияния z-переменной выражается пороговым значением веса $\left|\mathrm{c}_{\mathrm{kj}}\right| \geq \mathrm{c}(\mathrm{j}), \mathrm{k \epsilon}\{1, \ldots, \mathrm{n}\}$ для j-ой у-

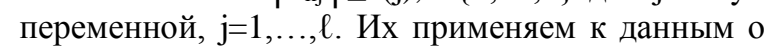
процессах обучения школьников в муниципальных школах США. В соответствии с нашей целью «что-то увидеть в данных» в [20] использован «когнитивный подход в моделировании, ориентированный на то, чтобы активизировать интеллектуальные процессы исследователя (субъекта) и помочь ему зафиксировать свое представление проблемной ситуации в виде формальной модели». Методология моделирования в [20] основана на моделировании субъективных представлений экспертов о ситуащии и включает модель представления знаний эксперта в виде ориентированного орграфа (когнитивной карты $[(\mathrm{Z}, \mathrm{Y}), \mathrm{C}]$, где $\left(\mathrm{Z}_{\mathrm{mn}}, \mathrm{Y}_{\mathrm{mn}}=\mathrm{Z}_{\mathrm{mn}} \mathrm{C}_{\mathrm{nn}}\right)$ - множество факторов (n Z- и n у-переменных) ситуации, $\mathrm{C}_{\mathrm{nn}}{ }^{-}$ множество измерений $\mathrm{n}^{2}$ причинно-следственных отношений между факторами ситуации). Здесь же используем оператор ИИ к 2 фактам, 3 правилам. Наша анализируемая таблица чисел $\mathrm{C}_{66}$ уменьшается до 2 столбцов, т.е. анализируем только 2 столбца ее : $\mathrm{C}_{\mathrm{n} 2}$.

Если выражаться простым языком, то значение у-переменной $\mathrm{y}_{\mathrm{ij}}$ равно значению $\mathrm{z}_{\mathrm{i} 1}$ и вес этой переменной равен $\mathrm{c}_{1 \mathrm{j}}$. например, в нашем примере (см.ниже $\mathrm{y}_{\mathrm{i} 1}=\mathrm{z}_{\mathrm{i} 2}(0.1790)+\mathrm{z}_{\mathrm{i} 3}(0.4803)+\ldots+\mathrm{z}_{\mathrm{i} 6}(0.4816)$. Из этих весов мы рассматриваем только значимые, т.е. рассматриваем те веса, абсолютное значение которого больше 0.48. Это позволит нам иметь дело только с теми весами и с названиями $\mathrm{z}-$ переменных, которые значимы. Остальные веса мы обнуляем при интерпретации - их вклад в значение $\mathrm{z}$-переменной (с номерами 1 и 4) считаем малым. Здесь мы применили правило: $\left|\mathrm{c}_{1 \mathrm{j}}\right| \geq 0.48$. Ниже убедимся, что таких весов в 1ой у-переменной 4 , их номера равны 2,3,5,6. А во второй: $\mathrm{c}_{12}$ и с $\mathrm{c}_{42}$.

Для наглядности мы построили граф связей [12] между одной $\mathrm{y}$-переменной и $\mathrm{z}$ переменными, значимо влияющими на эту $\mathrm{y}$ переменную. Для факта 1: $\lambda_{1}=3.6258$, назначим пороговое значение с (1), его значение полагаем (субъективно) равным 0.48 .

Мы конструировали [12] выводы по 3 правилам, по Факту 1. С применением причинноследственной зависимости в виде функции

$y_{i j}=z_{i 1} c_{1 j}+z_{i 2} c_{2 j}+\ldots+z_{i n} c_{n 1}, i=1, \ldots, m$, $\mathrm{j}=1,2$, (при $\mathrm{j}=1$ имеем у $\mathrm{i} 1=0.4803 \mathrm{z}_{\mathrm{i} 2}+0.4982 \mathrm{z}_{\mathrm{i} 3}$ $\left.+0.4805 \mathrm{z}_{\mathrm{i} 5}+0.4816 \mathrm{z}_{\mathrm{i} 6}+\varepsilon_{1}\right)$, где некоторые из совокупности измеренных (в числах) воздействий $\left(\mathrm{c}_{\mathrm{j} 1}, \mathrm{c}_{2 \mathrm{j}}, \ldots, \mathrm{c} \text { nj }\right)^{\mathrm{T}}$ на 1-ую новую переменную системы (по своему критерию) обнуляем [20]. В ее формуле присутствуют только те переменные $\mathrm{Z}_{\mathrm{ik}}$, которые имеют ненулевые веса с $\mathrm{kj} \neq 0$, выражающие отношения между факторами ситуации. Здесь $\ell=2$, ибо $\lambda_{2}=1.3953>1$ по критерию Кайзера-.Дикмана. Наш выбор критерия связан с нашей возможностью придать 
смыслы двум доминирующим по величине дисперсии у-переменным.

В У-переменной №1 с наибольшей вариабельностью, равной $\lambda_{1}=3.6258$, пороговое значение с(1) полагаем (субъективно) равным 0.4 [20]. Тогда значения $\mathrm{c}_{\mathrm{k} 1}$ по модулю превышают пороговое значение $\mathrm{c}(1)=0.48$, соответственно только 4 (из 6) z-переменных с номерами $\mathrm{k}=2,3,5,6$. Они оказывают «ощутимое» влияние на у-переменную №1 (в том числе преимущественно обеспечивают дисперсию $\lambda_{1}=3.6258$-наибольшую информативность, пропорциональную дисперсии) посредством «весов» $\quad \mathrm{c}_{21}=+0.4803, \mathrm{c}_{31}=+0.4982, \mathrm{c}_{51}=+0.4805$, $\mathrm{c}_{61}=+0.4816$.

Аналогично введем факты и правила для новой переменной №2. Орграфы, если их изобразить на рисунке, принадлежат одной многослойной проблемной области. Дуги (с «весами» со знаком "минус") орграфа имеют противоположное направление, чем дуги (с «весами» со знаком “плюс") орграфа на рисунке 1 [12]. Для 2-ой у-переменной с вариабельностью, равной $\lambda_{2}=1.3953$, полагаем субъективно пороговое значение с(2) на веса соответствующим умеренной связи и равным по модулю 0.65 , ибо при к=1 и к=4 значения $\mathrm{c}_{12}$ и $\mathrm{c}_{42}$ по модулю превышают пороговое значение $\mathrm{c}(2)=0.65$. Следовательно, только 2 zпеременные, характеризуют влияние школы и учителей на 2-ую $\mathrm{y}$ - переменную. Так zпеременная школа (с номером к=1) оказывает ощутимое влияние посредством «веса» $\mathrm{c}_{12}=$ 0.6548 на 2-ую новую переменную - «средняя оценка за устную речь в школе 6-классников», выставленную оплачиваемыми школой учителями («в расчете на одного школьника»). Средняя школьная оценка за устную речь школьника, выставленная оплаченными школой учителями оказывает ощутимое влияние (в направлении, противоположном, чем в новой переменной №1) посредством веса $\mathrm{c}_{42}=-0.7014$. Для новой переменной №2 аналогично вышеизложенному понимается совокупность всех прямых влияний весов $\mathrm{c}_{\mathrm{k} 2}, \mathrm{k}=1,4$, удовлетворяющих Правилу 2 «ощутимости»: $\mid \mathrm{c}_{\mathrm{kj}}$ $\mid \geq \mathrm{c}(2)$.

\section{Скрытые факторы индивидуального сознания}

Индивидуальное сознание есть отражение общественного бытия отдельным человеком через призму конкретных условий его жизни и его психологических особенностей. Это значит, что в сознании индивида сосуществуют (в одних случаях гармонично сочетаясь друг с другом, а в других-находясь в антагонистических противоречиях) различные духовные пласты, верования другие элементы. Индивидуальное сознание-своеобразный сплав общего, особенного и единичного в сознании личности.

Наше объяснение фактов и правил для модуля извлечения знаний ЭС из данных совокупность смыслов, подчиняющихся правилам для цифровых фактов, в том числе вычисленных с применением прямой и обратной моделей главных компонент [11,18]. Такому «осмыслению» подвергаются сами элементы матрицы $\Lambda, \mathrm{C}, \mathrm{Y}$ этой теории [12].

Четыре главные компоненты: обозначим их $\mathrm{y}_{1}, \mathrm{y}_{2}, \mathrm{y}_{3}, \mathrm{y}_{4}$ полагаем модельно некоррелированными, а соответствующие им факторы индивидуального сознания независимыми. Поставим в соответствие этим 4 не измеряемым показателям (обобщенным математическим факторам) теоретические случайные величины $\xi_{1}, \xi_{2}, \xi_{3}, \xi_{4}$. Значения показателей индивидуального сознания имеют размерности. Их идентификацию проведем в другой статье.

Наличие единицы измерения не удобно при делении или умножении 2-х величин разных размерностей. Например, значению величины х, равной произведению: $\mathrm{x}=6$ га $\times 10$ человек 7 тракторов $\times 1$ га невозможно назначить название. Лучше иметь дело с безразмерными величиной типа $\mathrm{x}=6 \times 10 \backslash 7 \times 1$. Результирующее i-ое значение $\mathrm{j}$-го показателя $\mathrm{x}_{\mathrm{ij}}{ }_{\mathrm{ij}}$ равно сумме 2-х слагаемых: $\mathrm{x}_{\mathrm{ij}}^{0}=\mathrm{z}_{\mathrm{ij}} \mathrm{S}_{\mathrm{j}}+\mathrm{x}_{\mathrm{j}}{ }^{\mathrm{cp}}$, $\mathrm{j}=1, \ldots, \mathrm{n}, \quad \mathrm{i}=1, \ldots, \mathrm{m}, \quad$ и имеет конкретную размерность. Переменная величина $\mathrm{z}_{\mathrm{ij}}=\left(\mathrm{x}_{\mathrm{ij}}^{0}-\mathrm{x}_{\mathrm{j}}^{\mathrm{cp}}\right) / \mathrm{s}_{\mathrm{j}}$ очищена от размерности, она является стандартизованной переменной. Моделирование $\mathrm{z}$-переменной независимо от средней $\mathrm{x}^{\mathrm{cp}}$ и дисперсии $\mathrm{s}^{2}$ позволяет придать сумме 2-х слагаемых: $\mathrm{x}_{\mathrm{ij}}^{0}=\mathrm{z}_{\mathrm{ij}} \mathrm{s}_{\mathrm{j}}+\mathrm{x}_{\mathrm{j}}{ }^{\mathrm{cp}}, \mathrm{j}=1, \ldots, \mathrm{n}, \mathrm{i}=1, \ldots, \mathrm{m}$, заранее заданные свойства: среднее арифметическое значение $\mathrm{j}$-ой $\mathrm{x}^{0}$-переменной равно $\mathrm{x}^{\mathrm{cp}}{ }_{\mathrm{j}}$, дисперсия $\mathrm{j}$-ой $\mathrm{x}$ - переменной равна $\mathrm{s}^{2}$. Модельные $\mathrm{z}$-переменные позволяют нам формализовать динамику изменений значений zпеременных, их взаимосвязи. А взаимосвязи между парами $\mathrm{z}$-переменных-выборочные коэффициенты корреляции, смоделировать в точности равными заданным значениям. Последние значения могут быть такими, какими они являются у реальных значений $\mathrm{z}$ переменных, линейные комбинации которых образуют наши 4 у-переменные $\mathrm{y}_{1}, \mathrm{y}_{2}, \mathrm{y}_{3}, \mathrm{y}_{4}$. После окончание этапа моделирования мы присвоим единицы измерения каждому из zпеременных, веса при которых имеют абсолютные значения, превышающие пороговые значения $\mathrm{c}^{0}(\mathrm{j}), \mathrm{j}=1,2,3,4$. 


\section{Алгоритм моделирования значений показателей индивидуального сознания}

Алгоритм состоит из 4 шагов. На 0-ом шаге, если $\mathrm{n}>4$, в дополнение к 4 собственным числам моделируем недоминирующие элементы.

Далее, имея полный спектр реализуем модель C.P.Chalmers-a[5]: $\Lambda_{\mathrm{nn}}=>\left(\mathrm{C}^{(\ell)}{ }_{\mathrm{nn}}, \mathrm{R}^{(\ell)}{ }_{\mathrm{nn}}\right), \mathrm{n}=4$, $\ell=1, \ldots, \mathrm{k}_{\ell}$. Из $\mathrm{k}_{\ell}$ штук матриц $\mathrm{C}^{(\ell)}{ }_{\mathrm{nn}}$ весов отбираем только те матрицы, у которых выделенные элементы удовлетворяют критерию 2. Получаем уравнения для переменных $\mathrm{y}_{1}, \mathrm{y}_{2}, \mathrm{y}_{3}, \mathrm{y}_{4}$ :

$$
\mathrm{y}_{\mathrm{i} 1}=\mathrm{z}_{\mathrm{i} 1} \mathrm{c}_{11}+\ldots+\mathrm{z}_{\mathrm{i} 4} \mathrm{c}_{41}, \mathrm{y}_{\mathrm{i} 2}=\mathrm{z}_{\mathrm{i} 1} \mathrm{c}_{12}+\ldots+\mathrm{z}_{\mathrm{i} 4} \mathrm{c}_{42}
$$
$\mathrm{y}_{\mathrm{i} 3}=\mathrm{z}_{\mathrm{i} 1} \mathrm{c}_{13}+\ldots+\mathrm{z}_{\mathrm{i} 4} \mathrm{c}_{43} ., \mathrm{y}_{\mathrm{i} 4}=\mathrm{z}_{\mathrm{i} 1} \mathrm{c}_{14}+\ldots+\mathrm{z}_{\mathrm{i} 4} \mathrm{c}_{44} \mathrm{i}=1, \ldots, \mathrm{m}$.

Обратная спектральная задача (ОС3[11]): $\Lambda_{\mathrm{nn}}=>\left(\mathrm{C}^{(\ell)}{ }_{\mathrm{nn}}, \mathrm{R}^{(\ell)}{ }_{\mathrm{nn}}\right), \ell=1, \ldots, \mathrm{k}_{\ell}, \quad$ реалиизующая алгоритм из работы [21]. В формулировке ОС3 применяется геометрический объект-конус, в ПСЗ-гиперэллипсоид. Отличие ПСЗ от ОС3 в том, что в ОСЗ моделируются і-ые компоненты $(\mathrm{i}=1, \ldots, \mathrm{n})$ всех $\mathrm{n}$ собственных векторов, т.е. моделируются строки $\mathbf{c}_{\mathbf{i}}=\left(\mathrm{c}_{\mathrm{i} 1}, \ldots, \mathrm{c}_{\mathrm{in}}\right), \mathrm{i}=1, \ldots, \mathrm{n}$, матрицы $\mathrm{C}_{\mathrm{nn}}$ (они имеют номер $\ell=1, \ldots, \mathrm{k}_{\ell}<\infty$ ). Компоненты вектор-строки сі. интерпретируются как координаты $\mathrm{n}$ точек на одной (из бесконечного числа) образующей конуса $\boldsymbol{K}_{\mathrm{nn}}[21]$. В проекции на плоскость, перпендикулярную основанию конуса (на плоскость, проходящую через вершину конуса перпендикулярно основанию конуса). При этом на боковой поверхности конуса получаются 2 прямые - 2 образующих конуса. По Лемме из [21] угол между ними равен $\pi / 2$. За одно обращение к программе CORMAT [22] алгоритма моделируются $\mathrm{n}$ образующих конуса, а на каждой образующей моделируются $\mathrm{n}$ точек с координатами $\mathrm{c}_{\mathrm{i} 1}, \ldots, \mathrm{c}_{\mathrm{in}}, \mathrm{i}=1, \ldots, \mathrm{n}$. Из этих $\mathrm{n}^{2}$ чисел образуется модельная матрица $C^{(\ell)}{ }_{66}$, У которой мы анализируем только 4 первых столбца. Из матриц $\mathrm{C}^{(\ell)}{ }_{66} \quad \mathrm{c}$ номером $\ell=1, \quad \ldots, \mathrm{k}_{\ell}<\infty, \quad$ если мы моделируем $\mathrm{k}_{\ell}=200$ матриц $\mathrm{C}^{(\ell)}$ n. Ее j-ый столбец интерпретируется как вектор-столбец собственного вектора, зависящего от собственного числа $\lambda_{\mathrm{j}} \quad \mathrm{j}=1, \mathrm{n}, \quad \mathbf{c}_{\mathrm{j}} \Lambda_{66} \mathbf{c}^{\mathbf{T}}{ }_{\mathrm{j}}$ $=1, \mathbf{c}_{\mathrm{j}}=\left(\mathrm{c}_{1 \mathrm{j}}, \ldots, \mathrm{c}_{\mathrm{nj}}\right)^{\mathrm{T}}, \quad \mathrm{j}=1, \ldots, 6$. Эти равенства показывают зависимость $\mathrm{j}=$ го собственного вектора (вектора «весов») от всех собственных чисел. В ОС $3: \Lambda_{\mathrm{nn}}=>\left(\mathrm{C}^{(\ell)}{ }_{\mathrm{nn}}, \mathrm{R}^{(\ell)}{ }_{\mathrm{nn}}\right), \quad \ell=1, \ldots, \mathrm{k} \ell$, компьютерную программу CORMAP решения которой мы применяем $\left(\mathrm{k}_{\ell}=200\right)$, моделируются не $\mathrm{n}$ компонент j-ых собственных векторов $\mathbf{c}_{\mathrm{j}}=\left(\mathrm{c}_{1 \mathrm{j}}, \ldots, \mathrm{c}_{\mathrm{nj}}\right)^{\mathrm{T}}$, а i-ые компоненты $(\mathrm{i}=1, \ldots, \mathrm{n})$ всех $\mathrm{n}$ собственных векторов, т.е. моделируются строки $\mathbf{c}_{\mathrm{i} .}=\left(\mathrm{c}_{\mathrm{i} 1}, \ldots, \mathrm{c}_{\mathrm{in}}\right), \mathrm{i}=1, \ldots, \mathrm{n}$, матрицы $\mathrm{C}_{\mathrm{nn}}$. Так как номер компоненты собственного вектора $\mathbf{c}_{\mathrm{j}}=\left(\mathrm{c}_{1 \mathrm{j}}, \ldots, \mathrm{c}_{\mathrm{nj}}\right)^{\mathrm{T}}$ равен номеру $\mathrm{z}$-переменной, то в OC3 моделируются последова тельно значения весов $\left(\mathrm{c}_{11}, \ldots, \mathrm{c}_{1 \mathrm{n}}\right)$. Сперва моделируются веса $\left(\mathrm{c}_{11}, \ldots, \mathrm{c}_{1 \mathrm{n}}\right)$ с учетом всех значений $\lambda_{1}, \ldots, \lambda_{\mathrm{n}}$ весов, затем моделируются веса $\left(c_{21}, \ldots, c_{2 n}\right)$ с учетом всех значений $\lambda_{1}, \ldots, \lambda_{n}$ весов, и на n-ом шаге моделируются веса $\left(c_{n 1}, \ldots, c_{n n}\right)$ с учетом всех значений $\lambda_{1}, \ldots, \lambda_{\mathrm{n}}$ весов. Это означает, что на каждом шаге моделирования весов сохраняется соответствие номера i z-переменной к ее смыслу. Программа CORMAP соответствует нашей модели, потебовалась лишь модификация по применению Критериев 1-4. Смысл і-ой zпеременной неявно участвует при нашем моделировании матрицы весов. Аналитику остается лишь выявить названия и смыслы Zпеременных, опираясь на внешние источники информации [1-9]. Но это требует логики высокого качества от эксперта. Наши эксперименты показали: последовательность шагов нахождения не совпадает с последовательностью вычисления весов возможно нахождение сперва смысла Zпеременной с большим номером, потом - c меньшим номером.

Применим интерпретации компонент собственных векторов неизвестной корреляционной матрицы с заданным спектром $\Lambda_{\mathrm{nn}}=\operatorname{diag}\left(\lambda_{1}, \ldots \lambda_{\mathrm{n}}\right), \mathrm{n}=6$. При этом в ОС3 значения собственных чисел (и в интерпретируются нами здесь и в работах [11-21] как длины полуосей гиперэллипсоида, а компоненты собственных векторов - как косинусы (синусы) углов между i-ой zпеременной и j-ой $\mathrm{y}$-переменной $\mathrm{c}_{\mathrm{ij}}=\operatorname{corr}\left(\mathrm{z}_{\mathrm{i}}, \mathrm{y}_{\mathrm{j}}\right)$. Необходимо моделировать и анализировать значения парных коэффициентов корреляции Пирсона, как показано ниже, двух видов: $\mathrm{r}_{\mathrm{ij}}=\operatorname{corr}\left(\mathrm{z}_{\mathrm{i}}, \mathrm{Z}_{\mathrm{j}}\right), \quad \mathrm{c}_{\mathrm{ij}}=\operatorname{corr}\left(\mathrm{Z}_{\mathrm{i}}, \mathrm{y}_{\mathrm{j}}\right), \quad$ и одну дисперсию $\lambda_{\mathrm{j}}=\operatorname{corr}\left(\mathrm{y}_{\mathrm{j}}, \mathrm{y}_{\mathrm{j}}\right)$. При этом парный коэффициент корреляции Пирсона $\mathrm{r}_{\mathrm{ij}}$ служит коэффициентом линейной связи между к-ими значениями i-ой $\mathrm{z}-$ переменной и j-ой $\mathrm{z}$-переменной: $\quad \mathrm{z}_{\mathrm{ki}}=\mathrm{r}_{\mathrm{ij}} \times \mathrm{z}_{\mathrm{kj}}$. $\mathrm{k}=1, \ldots, \mathrm{m}, \quad \mathrm{i}=1, \ldots, \mathrm{n}, \quad \mathrm{j}=1, \ldots, \mathrm{n}$. Эта формула позволяет вычислить значения i-ой z-переменной с неизвестным смыслом через значения j-ой zпеременной с известным смыслом, что облегчает процесс присваивания смыслов всем $\mathrm{n}$ Zпеременных.

\section{Пример присвоения имен значениям показателям индивидуального сознания}

Зафиксируем спектр $\Lambda_{66}=\operatorname{diag}(2.5000,1.0000$, $1.0000, \quad 1.0000,0.3000,0.2000)$. Экспертно согласованными значениями являются параметры спектра: Если имеем матрицу $\mathrm{R}_{\mathrm{nn}}$ или ее спектр $\Lambda_{\mathrm{nn}}$, то значения их вычисленных f-параметров попадают всегда внутрь своих интервалов изменения $\quad[2,6,7]: \quad \mathrm{f}_{1}\left(\Lambda_{\mathrm{nn}}\right) / \mathrm{n} \leq \mathrm{f}_{2}\left(\Lambda_{\mathrm{nn}}\right) \leq \mathrm{f}^{2}{ }_{1}\left(\Lambda_{\mathrm{nn}}\right)$, $\mathrm{f}^{2}{ }_{1}\left(\Lambda_{\mathrm{nn}}\right) \geq \mathrm{n} \times \mathrm{f}^{\left(2 /{ }^{\mathrm{n}}\right)}{ }_{2}\left(\Lambda_{\mathrm{nn}}\right), \quad \mathrm{f}_{3}\left(\Lambda_{\mathrm{nn}}\right) \times \mathrm{f}_{5}\left(\Lambda_{\mathrm{nn}}\right) \leq \mathrm{f}^{(\mathrm{n} / 2)}{ }_{2}\left(\Lambda_{\mathrm{nn}}\right)$, $\mathrm{f}_{6}\left(\Lambda_{\mathrm{nn}}\right) \geq(\mathrm{n}-1) \times \mathrm{f}_{3}\left(\Lambda_{\mathrm{nn}}\right)^{1 /(\mathrm{k}-1)}, \quad 0 \leq \mathrm{f}_{4}\left(\Lambda_{\mathrm{nn}}\right) \leq 1 . \quad$ будем 
использовать формулы зависимости дисперсии іго вектора весов от взаимного расположения собственных чисел $\lambda_{\mathrm{i}}, \mathrm{i}=1, \ldots, \mathrm{n}$, друг от друга [11]:

$$
\operatorname{var}\left(c_{i}\right) \leq \frac{1}{m} \sum_{\substack{j=1 \\ j \neq i}}^{n} \frac{\lambda_{j} / \lambda_{i}}{\left(1-\lambda_{j} / \lambda_{i}\right)}
$$

Эта оценка выведена для спектра $\Lambda_{\text {nn }}$ выборочной корреляционной матрицы $\mathrm{R}_{\mathrm{nn}}$ многомерной выборки $Z_{\mathrm{mn}}$ из многомерного нормального распределения [ 11].

Задачи задания доминирующих значений и вычисления недоминирующих элементов спектра $\Lambda_{66}$ неизвестных корреляционных матриц $\mathrm{R}^{(\ell)}{ }_{66}, \ell=1, \ldots, \infty$, с заданными или неизвестными значениями наборов f-параметров видов $\left(\mathrm{f}_{3}, \mathrm{f}_{6}\right)$, $\left(f_{1}, f_{5}\right),\left(f_{3}, f_{4}, f_{6}\right),\left(f_{1}, f_{2}, f_{5}\right),\left(f_{1}, f_{2}, f_{4}\right),\left(f_{1}, f_{3}, f_{4}, f_{6}\right),\left(c \times f_{1}, c^{2} \times f_{2}\right.$,

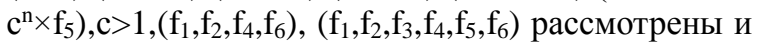
решены в работах [11-17]. Для каждой корреляционной $\quad$ матрицы $\quad \mathrm{R}^{(\ell)}{ }_{n n}, \ell=1, \ldots, \infty$, получены модельные многомерные $\Lambda$-выборки $\mathrm{Y}^{(\mathrm{t})}{ }_{\mathrm{mn}} \in N_{s}(0, \Lambda)$, многомерные R-выборки $\mathrm{Z}^{(\mathrm{t}, \ell)}{ }_{\mathrm{mn}} \in$ $\boldsymbol{N}_{s}\left(0, \mathrm{R}^{(\ell)}\right), \quad \mathrm{t}=1, \ldots \mathrm{k}_{\mathrm{t}}, \quad \ell=1, \ldots, . \quad \mathrm{k}_{\ell}$. Случайная nмерная выборка объема $\mathrm{m}>\mathrm{nU}^{(\mathrm{t})}{ }_{\mathrm{mn}} \in \boldsymbol{N}_{s}\left(0, \mathrm{I}_{\mathrm{nn}}\right)$, номер $\mathrm{t}=1, \ldots \mathrm{k}_{\mathrm{t}}<\infty$, с выборочной корреляционной матрицей $\mathrm{I}_{\mathrm{nn}}$ и $\mathrm{c}$ неизвестным законом распределения с помощью случайных линейных преобразований превращается в многомерные выборки $\quad \mathrm{Y}^{(\mathrm{t})}{ }_{\mathrm{mn}} \in \boldsymbol{N}_{s}(0, \Lambda), \quad \mathrm{Z}^{(\mathrm{t}, \ell)}{ }_{\mathrm{mn}} \in \boldsymbol{N}_{s}\left(0, \mathrm{R}^{(\ell)}\right)$, $\mathrm{t}=1, \ldots \mathrm{k}_{\mathrm{t}}, \quad \ell=1, \ldots, . \quad \mathrm{k}_{\ell} . \quad$ При всех наборах $\mathrm{f}-$ параметров спектра $\Lambda$ локазаны гистограммная и модельная адекватности $(\mathrm{R})-,(\mathrm{C}, \Lambda)-,(\mathrm{C}, \Lambda, \mathrm{Y})-$, выборок реальным выборкам разного объема и числа п переменных [23-25].
Для нашего спектра $\Lambda_{66}=\operatorname{diag}(2.5000,1.0000$, $1.0000,1.0000,0.3000,0.2000)$ реализуем вариант №3 OM $\quad$ ОК: $\quad \Lambda_{66}=>\left(\mathrm{C}^{(\ell)}{ }_{66}, \mathrm{R}^{(\ell)}{ }_{66}, \mathrm{Y}^{(\mathrm{t})}{ }_{20,6}, \mathrm{Z}^{(\mathrm{t}, \ell)}{ }_{20,6}\right)$, $\mathrm{t}=1, \ldots \mathrm{k}_{\mathrm{t}}, \ell=1, \ldots, \mathrm{k}_{\ell}$. Значения $\mathrm{f}_{1}=6, \mathrm{f}_{2}=9,38, \mathrm{f}_{3}=12.5$, $\mathrm{f}_{4}=0.916666667, \mathrm{f}_{5}=0.15, \mathrm{f}_{6}=9,333333333$. Значение одного из главных f-параметров спектра $\mathrm{f}_{4}=0.916666667$ свидетельствует о том, что значения дисперсий 4-х факторов отражают 92\% информации, содержащихся в 4-х у-переменных или в 6 z-переменных. Восемь процентов (8\%), содержащихся в 2-х неучтенных у-переменных, наша модель, наше когнитивное моделирование не использует, из-за ограниченности индивидуального восприятия индивида только 4мя факторами. Анализ значений других $\mathrm{f}$ параметров аналогичен приведенным в [11-17].

Модельные эксперименты проводились при разных значениях $\mathrm{k}_{\mathrm{t}}, \mathrm{k}_{\ell}$. Формировались и анализировались виртуальные базы данных [14]. Исследования продолжаются. Здесь не будем анализировать, интерпретировать элементы выборки $Z^{(t, \ell)} 20,6$, их результаты будут опубликованы отдельно. Ограничимся анализом только присвоений смыслов Z-переменным, имеющим умеренную степень $(\operatorname{const}(\mathrm{j})=0.5191$, $\mathrm{j}=1,2,3,4)$ своих «весов». Выбор этого критерия связан с нашей возможностью придать смыслы Zпеременным, влияющих на 4 доминирующие по величине дисперсий у-переменные.

Рассмотрим матрицу весов $\mathrm{C}^{(\ell)} 66$ Таблица 1 .

\section{Матрица весов $\mathbf{C}^{(\ell)}$}

Таблица 1

\begin{tabular}{|l|l|l|l|l|l|l|}
\hline ROW 1 & .4971 & -.3084 & $\mathbf{- 0 . 5 7 3 9}$ & -.2884 & -.1500 & -.4720 \\
\hline ROW 2 & .0736 & -.4618 & -.0668 & .3923 & -.6613 & .4309 \\
\hline ROW 3 & .4481 & -.3032 & -.0492 & .4155 & .6819 & .2592 \\
\hline ROW 4 & .2227 & $-\mathbf{0 . 6 3 0 3}$ & .2150 & -.6420 & .1770 & .2519 \\
\hline ROW 5 & $\mathbf{0 . 5 1 9 1}$ & .4397 & -.0281 & -.4220 & -.1139 & .5876 \\
\hline ROW 6 & .4771 & -.0953 & $\mathbf{0 . 7 8 5 4}$ & .0093 & -.1756 & -.3399 \\
\hline
\end{tabular}

$\operatorname{corr}\left(\mathrm{y}_{1}, \mathrm{z}_{5}\right)=0.5191 \geq \operatorname{const}(1)$

корреляция между 1-ой y-переменной и 5-ой $\mathrm{z}$-переменной выражена умеренно: $\operatorname{corr}\left(\mathrm{y}_{1}, \quad \mathrm{z}_{5}\right)=0.5191$, корреляция между 1-ой у-переменной и 5-ой zпеременной, то смысл этой переменной придадим после обсуждения всех выраженных умеренно переменных. Анализ всех «весов» выявил следующую картину. В уравнении для 2-ой переменной $\mathrm{y}_{2}$ «вес» $\mathrm{c}_{42}$ имеет умеренную степень корреляции - $\operatorname{corr}\left(\mathrm{y}_{2}, \mathrm{z}_{4}\right)=-.6303$ $\geq 0.5191 \geq$ const $(2)$. В уравнении для 3-ей переменной $\mathrm{y}_{3}$ веса $\quad \mathrm{c}_{13}=-0.5739, \mathrm{c}_{63}=-0.7854$ имеют умеренную и более умеренную степени корреляции $\quad-\left|\mathrm{c}_{13} \quad\right| \geq \operatorname{const}(3), \quad\left|\mathrm{c}_{63}\right| \geq \operatorname{const}(3) . \quad$ В уравнении для 4-ой у-переменной у4 вес $\mathrm{c}_{14}$ имеет умеренную степень корреляции - $\mathrm{c}_{64}=-$ .6420, $\left|\mathrm{c}_{64}\right| \geq \operatorname{const}(4)$.

Приступим к присваиванию имен к каждой из выделенных z-переменных. Так как смысл упеременной у означает «по правде (правильно) поступать», то $\mathrm{z}$-переменную $\mathrm{z}_{5}$, (достаточно сильно коррелирующей с у-переменной $\mathrm{y}_{1}$ ), при своим смысл «честность». Так как смысл у-пере менной у 2 означает «принудить к...», и «вес».7854 имеет знак, противоположный знаку «веса» $\operatorname{corr}\left(\mathrm{y}_{1}, \mathrm{z}_{5}\right)=0.5191$, то $\mathrm{z}-$-переменной $\mathrm{z}_{6}$ припишем смысл «бывшый партократ». Смысл Zпеременной $\mathrm{z}_{1}$ в «весом» -0.5739 припишем 
«проявление командного стиля общения ...». Так как смысл у-переменной уз означает «обмануть, чтобы...», то z-переменную $\mathrm{z}_{1}$ припишем смысл «лицо низкой социальной активности», а Zпеременной $\mathrm{Z}_{2}$-смысл «проявление некомпетентности».

Так как смысл у-переменной у4 означает «страху нагнать», то Z-переменной $\mathrm{z}_{4}$ припишем смысл «проявление деспотизма».

Целенаправленные чтения текстов работ [19] убедили нас в правильности применении когнитивного [20] восприятия ощущений, восприятий, реакций индивида на принуждение, на «свои страхи», на «обмануть, чтобы...». Не учитывались в нашей Анализ проводился с применением языка описания ситуаций показатель- наименование - значение - единица измерения». В этой статье реализованы первые 2 возможности языка описания ситуаций. Реализации двух остальных возможностей «языка» будут описаны в отдельной статье.

\section{Заключение}

Наша цель состояла в том, чтобы показать возможности применения способа описания ситуаций «показатель-наименование-значениеединица измерения», когнитивного моделирования взаимосвязей между измеряемыми показателями индивида и скрытыми неизмеряемыми независимыми факторами воздействия на индивиды разработать модель и получить экспертным путем, руководствуясь только цифровыми фактами: $\lambda_{1}=2.5000, \quad \lambda_{2}=1.0000, \quad \lambda_{3}=1.0000, \quad \lambda_{4}=1.0000$, $\lambda_{5}=0.3000, \quad \lambda_{6}=0.2000$ и Правилами $1,2,3,4$, получить названия заметных показателей, достаточно тесно связанных с скрытими факторами воздействия на индивида. Мы обращаем внимание на полезность применения аппарата системы объяснений фактов и правил для модуля извлечения знаний из данных, при формулировке содержательных выводов при интерпретации цифровых результатов применения модели главных компонент в плохо формализуемой науке-индивидуальное сознание. Разработанная математическая модель цифровизации показателей индивидуального сознания индивида правдоподобно выявляет части элементов языка описания ситуаций «показатель - наименование - значение - единица измерения». Когнитивный анализ и когнитивное моделирование демонстрировали эффектвность применения Обратной Модели Главных Компонент [11]. Иллюстратиный пример и дискуссия по приданию названий 6 коррелированным показателям индивидуального сознания при заданных 4 скрытых факторах индивидуального восприятия служат обоснованием дальнеших исследований по моделированию значений и выбору единиц измерения $\mathrm{n}$ коррелированным показателям индивидуального сознания, оценкам их средних и дисперсий.

При этом практические решения по принятию предыдущего значения и последующего предыдущего значения и последующего предыдущего значения и подвергнуты всестороннему анализу. Чтобы осмыслить изучаемые в этой модели процессы необходимо рассмотреть вопросы при переходе от безразмерных значений z-переменных к значениям $\mathrm{x}^{0}$-переменных (измеренных значений показателей) с размерностями. Здесь предстоит имееть дело с значениями средних арифметических $\mathrm{n}$ зависимых переменных $\mathbf{x}^{\mathrm{cp}}$ $=\left(\mathrm{x}^{\mathrm{cp}}{ }_{1}, \ldots, \mathrm{x}_{\mathrm{n}}^{\mathrm{cp}}\right), \mathrm{c}$ их стандартными отклонениями, c эластичностями переменной $\mathrm{x}_{\mathrm{j}}$ по $\mathrm{x}$ переменным $\mathrm{x}_{\mathrm{i}} \neq \mathrm{x}_{\mathrm{j}}$, где $\mathrm{x}_{\mathrm{ij}}=\mathrm{x}_{\mathrm{ij}}^{0}-\mathrm{x}_{\mathrm{j}}^{\mathrm{cp}}, \mathrm{i}=1, \ldots, \mathrm{m}$, $\mathrm{j}=1, \ldots, n$. Это позволит оценивать приращение значения $\mathrm{x}_{\mathrm{n}}^{0}$ при заданном приращении значения одной независимой $\mathrm{x}^{0}$-переменной.

Предстоит исследовать обнаруженные новые явления, объекты, проявления их свойств и необходимо разрабатывать алгоритмические методы работы с ними, выходить на новые рубежи знаний «на личностном, индивидуальном уровне» на личностном, индивидуальном уровне [1], так как «массовое сознание остается в «домашних рамках» [1]. Мы надеемся, что данное направление исследований будет способствовать преобразованию индивидуального сознания к открытости и восприимчивости. «Открытость и восприимчивость к лучшим достижениям, а не заведомое отталкивание всего «не своего» - вот залог успеха и один из показателей открытого сознания» [1]. 


\begin{tabular}{|c|c|c|c|c|c|c|}
\hline Impact Factor: & $\begin{array}{l}\text { ISRA (India) } \\
\text { ISI (Dubai, UAE } \\
\text { GIF (Australia) } \\
\text { JIF }\end{array}$ & $\begin{array}{l}=1.344 \\
=0.829 \\
=0.564 \\
=1.500\end{array}$ & $\begin{array}{l}\text { SIS (USA) } \\
\text { PИНЦ (Russia) } \\
\text { ESJI (KZ) } \\
\text { SJIF (Morocco) }\end{array}$ & $\begin{array}{l}=0.912 \\
=0.207 \\
=4.102 \\
=2.031\end{array}$ & $\begin{array}{l}\text { ICV (Poland) } \\
\text { PIF (India) } \\
\text { IBI (India) }\end{array}$ & $\begin{array}{l}=6.630 \\
=1.940 \\
=4.260\end{array}$ \\
\hline
\end{tabular}

References:

1. Nazarbaev N.A. (2018) Vzglyad v budushchee: modernizatsiya obshchestvennogosoznaniya. www.akorda.kz/ru/ events/ akorda_news/press_conferences/statya-glavygosudarstva-vzglyad-v-budushcheemodernizaciya-obshchestvennogo-soznaniya

2. (2018) Mezhdunarodnaya konventsiya o likvidatsii vsekh form rasovoy diskriminatsii

3. (2018) Deklaratsiya OON o rase i rasovykh predrassudkakh

4. (2018) Evropeyskaya komissiya protiv rasizma i neter pimosti (angl.) (fr.)

5. Kon I. (2018) «Psikhologiya predrassudka»podrobnyy material o sotsial'nopsikhologicheskikh kornyakh etnicheskikh predubezhdeniy

6. Alen de Benua (2018) Chto takoe rasizm?//Ateney.-№5.-p. 21-26.

7. (2008) Kritika rasizma v sovremennoy Rossii i nauchnyy vzglyad na problemu etnokul'turnogo mnogoobraziya.-M:Moskovskoe byuro po pravam cheloveka, “Academia", 2008.- p. 124.

8. Davidson A.B. (2011) Antirasistskiy rasizm.Novaya i noveyshaya istoriya. Provereno 30 oktyabrya 2011. Arkhivirovano 1 sentyabrya 2012 goda.

9. N.Kevorkova. (2018) Universitety vyzhivaniya inostran nykh studentov v Rossii.

10. Mosteller F., T'yuki Dzh. (1982) Analiz dannykh i regressiya: v 2-kh vypuskakh, vyp. 2. - M.: Finansy i statistika, 1982. - 239 p.

11. Zhanatauov S.U. (2013) Obratnaya model' glavnykh kom ponent. - Almaty: Kazstatinform, 2013. - $201 \mathrm{p}$.

12. Zhanatauov S.U. (2013) Kognitivnaya karta i model' sotsial'no-ekonomicheskikh faktorov kar'ernoy uspeshnosti shkol'nikov munitsipal'nykh shkol SShA.Sibirskiy pedagogicheskiy zhurnal. 2013, №6, p. 28-33.

13. Zhanatauov S.U. (2015) Kognitivnaya karta i kognitivnaya model' analiza glavnykh komponent (telekom munikatsionnaya otrasl').Natsional'naya assotsia tsiya uchenykh (NAU).IX Mezhd. nauch.-prakt. konf: «Ote chestvennaya nauka $\mathrm{V}$ epokhu izmeneniy:postulaty proshlo go i teorii novogo vremeni».Rossiya, Ekate rinburg,16-17 maya 2015g. p. 55-58.

14. Zhanatauov S.U. (2014) Analiz budushchikh debitorskoy i kreditorskoy zadolzhennos tey munitsipalitetov gorodov.Ekonomicheskiy analiz:teoriya i praktika. M.:№2(353), 2014g., p.54-62. www.fin-izdat.ru/journal/ analiz/
15. Zhanatauov S.U. (2017) A model of calculation risk changing of the interest rate "yield to maturity date" for foreign currency bonds of the republic of Kazakhstan. Interna tional scientific journal Theore tical \& Applied Science. 2017,№ 8, vol. 52, p. 19-36. www.TScience.org

16. Zhanatauov SU. (2018) Inverse model of multiple linear regression analysis. International scientific journal Theoretical \&Applied Science.2018, №4(60):201-212. www.T-Science.org .

17. Zhanatauov SU. (2018) A model of calculation of subjective probabilities in business. Intern. scientific journal Theoretical\&AppliedScience. 2018, №5(61): p.142 -156.

18. Hotelling H. (1933) Analysis of a complex of statistical variables into principal components. J.Educ.Psych. ,1933,v.24, pp 417,441,498-520.

19. Zhanatauov SU. (2017) Theorem on the $\Lambda$ samples. Intern ational scientific journal Theoretical \&Applied Science. 2017,№ 9, vol. 53, p.177-192. www.T-Science.org .

20. Axelrod R. (1976) The Structure of Decision:Cognitive Maps of Political ElitesPrinceton.Univ.Press, 1976.

21. Chalmers C.P. (1975) Generation of correlation matrices with a given eigen-structure. -J. Stat. Comp. Simul., 1975, vol.4, p.133-139.

22. Zhanatauov S.U. (1988) Ofunktsional'nom napolnenii PPP "Spektr". Sistemnoe modelirovanie-13.-Novosi birsk, 1988, p.3-11.

23. Zhanatauov S.U. (2016) Model and histogram to adequacy of variables $(C, \Lambda)$-samples and real multidimensional sample. International Scientific Journal Theoretical\& Applied Science.2016,№ 11,vol. 43, p.53-61. www.TScience.org

24. Zhanatauov S.U. (2017) Modelirovanie mnogomernykh vyborok znacheniy priznakov zernovoy kul'tury. "II mezhdun. nauchnoprakt.konf. «Evropa i tyurkskiy mir: nauka,tekhnika i tekhnologii".Izmir (Turtsiya), 29-31 maya2017. www.region.academ.org .

25. Zhanatauov S.U. (2014) The (C, $\Lambda, Y)$-sample is adequate to real multidimensional sample. Proced. Intern. Sonf.. "Leadership in Education, Business and Culture". 25 apriel 2014,AlmatySeatle,ICET USA. Leadership Iternational Conference "Leadership on Education, Business and Culture». p.151-155. 